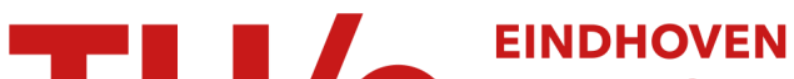 \\ UNIVERSITY OF \\ TECHNOLOGY
}

\section{Spin-up in a circular tank with a radical barrier}

Citation for published version (APA):

van de Konijnenberg, J. A., Wessels, T. L., \& Heijst, van, G. J. F. (1996). Spin-up in a circular tank with a radical barrier. Physics of Fluids, 8(8), 2048-2059. https://doi.org/10.1063/1.869007

DOI:

10.1063/1.869007

Document status and date:

Published: 01/01/1996

\section{Document Version:}

Publisher's PDF, also known as Version of Record (includes final page, issue and volume numbers)

\section{Please check the document version of this publication:}

- A submitted manuscript is the version of the article upon submission and before peer-review. There can be important differences between the submitted version and the official published version of record. People interested in the research are advised to contact the author for the final version of the publication, or visit the $\mathrm{DOI}$ to the publisher's website.

- The final author version and the galley proof are versions of the publication after peer review.

- The final published version features the final layout of the paper including the volume, issue and page numbers.

Link to publication

\section{General rights}

Copyright and moral rights for the publications made accessible in the public portal are retained by the authors and/or other copyright owners and it is a condition of accessing publications that users recognise and abide by the legal requirements associated with these rights.

- Users may download and print one copy of any publication from the public portal for the purpose of private study or research.

- You may not further distribute the material or use it for any profit-making activity or commercial gain

- You may freely distribute the URL identifying the publication in the public portal.

If the publication is distributed under the terms of Article 25fa of the Dutch Copyright Act, indicated by the "Taverne" license above, please follow below link for the End User Agreement:

www.tue.nl/taverne

Take down policy

If you believe that this document breaches copyright please contact us at:

openaccess@tue.nl

providing details and we will investigate your claim. 


\title{
Spin-up in a circular tank with a radial barrier
}

\author{
J. A. van de Konijnenberg, T. L. Wessels, and G. J. F. van Heijst \\ Fluid Dynamics Laboratory, Department of Technical Physics, Eindhoven University of Technology, \\ P.O. Box 513, $5600 \mathrm{MB}$ Eindhoven, The Netherlands
}

(Received 25 January 1996; accepted 17 April 1996)

\begin{abstract}
The time-dependent motion of fluid in a circular tank with a radial barrier as a result of an increase in angular velocity of the tank is investigated. The length of the barrier is considered as the main experimental parameter. The flow field immediately after the increase in angular velocity is calculated analytically. Experiments have been performed with a tank placed on a rotating table. Quantitative results for the time-dependent flow were obtained by the tracking of small particles floating at the free surface of the fluid. The flow appears to be characterized by separation from the end of the barrier and the subsequent formation of a stable vortex pattern. The trajectory of the vortex that is shed from the end of the barrier is determined with dye visualization, and compared with analytical results from a point-vortex model. () 1996 American Institute of Physics. [S1070-6631(96)01608-X]
\end{abstract}

\section{INTRODUCTION}

During the last decades, the spin-up of a homogeneous fluid to a final state of solid-body rotation with angular velocity $\Omega$ due to an increase $\Delta \Omega$ in the angular velocity of its container has received considerable attention. Most studies on this subject concerned the flow in a circular tank (Greenspan and Howard, ${ }^{1}$ Wedemeyer, ${ }^{2}$ Weidman, ${ }^{3}$ Hyun, ${ }^{4}$ Van de Konijnenberg and Van Heijst ${ }^{5}$ ). In a circular geometry, the flow remains approximately azimuthal, and slowly gains a higher angular velocity by a secondary flow driven by the Ekman layers at the bottom and the lid of the fluid. Van Heijst ${ }^{6}$ first studied spin-up in cylindrical geometries with non-circular cross section, such as a semi-circle and a circle with a radial barrier. This article was followed by a number of publications on spin-up in a number of noncircular geometries, most of them rectangular (Van Heijst, Davies and Davis, ${ }^{7}$ Van Heijst, Maas and Williams, ${ }^{8}$ Suh, ${ }^{9}$ Van de Konijnenberg ${ }^{10}$ ). It appeared that in almost all noncircular cylinders, the spin-up flow can be divided into a number of stages:

(1) Initial stage, characterized by a uniform relative vorticity and a shear layer at the boundaries.

(2) Separation stage; detachment from the sidewalls and formation of vortices.

(3) Organization stage; formation of a quasi-steady cellular pattern that fills the entire flow domain.

(4) Decay stage; the quasi-steady vortex pattern slowly spins up due to Ekman pumping.

The organization into a quasi-steady streamline pattern is highly sensitive to experimental parameters such as the shape of the geometry and the angular velocity. Geometries that can accommodate a small number of equally-sized vortices, such as a rectangular tank with aspect ratio $3: 1$, favour the formation of a quasi-steady vortex pattern. The selforganization phenomena in spin-up experiments are related to the two-dimensional nature of rotating flows. Spin-up flows are approximately horizontal and independent of the vertical coordinate, and therefore show a similar behaviour as flows in, e.g., soap films and in a stratified environment.
However, the presence of Ekman layers leads to differences with purely two-dimensional flows, in particular in late stages of the spin-up process.

The present paper deals with the spin-up of a freesurface fluid in a circular tank with a barrier partially along its diameter. The length of the barrier is the main parameter of this study. In view of the otherwise cumbersome formulae in this paper, this length is represented by the coordinates of the end point. Both a polar coordinate system $(r, \theta)$ and a Cartesian coordinate system $(x, y)$ are used, with the centre of the circular section as the origin. The radius of the circular part of the boundary is denoted by $a$, the end point of the radial barrier is denoted in Cartesian coordinates by $(p a, 0)$ (see the configuration on the left side in Fig. 1). Thus, the dimensionless parameter $p$ may vary between -1 (yielding a partition of the domain into two semi-circular regions) and 1 (a purely circular geometry). This choice of domain shape is motivated as follows. First, one can investigate in this way whether the properties of the flow observed in a rectangular tank [such as self-organization, evolution of $\omega(\psi)$-profiles] also appear in a totally different geometry. Second, in this geometry the flow separates from the end of the barrier. The fact that the separation point is well-defined makes it possible to use simple models for vortex shedding. Third, by taking a short barrier one obtains a geometry resembling a circular tank. Thus, one can study the response to the spin-up in a circular geometry to a small perturbation in the shape of the domain.

Some of these flows have been studied earlier: experimental results on the flow in a geometry with a barrier length equal to the radius of the tank $(p=0)$ were published by Van Heijst, ${ }^{6}$ and experimental and numerical results on the flow on a semi-circular domain $(p=-1)$ were published by Van Heijst ${ }^{6}$ and Andersson, Billdal and Van Heijst. ${ }^{11}$ The experimental results in those papers consist of qualitative images of particle trajectories. In this paper, quantitative data on the experimental velocity field are presented and the previous results are extended to intermediate barrier lengths.

In section III, analytical results for the starting flow as a function of $p$ are presented. Experimental results for spin-up 


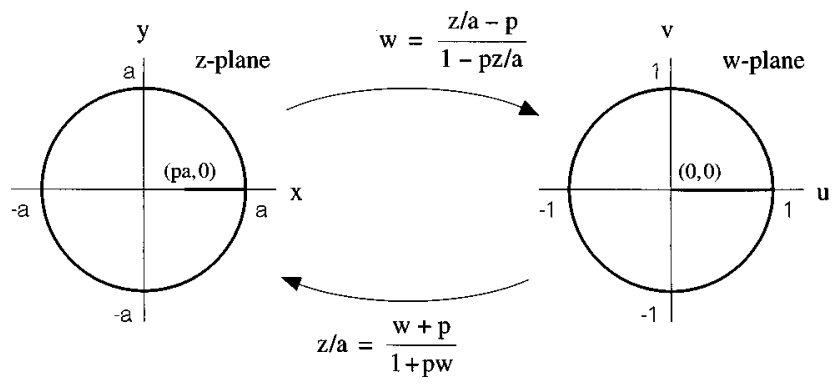

FIG. 1. Conformal mapping from the complex $z$-plane to the complex $w$-plane. By this mapping the geometry with the radial barrier ending at $(p a, 0)$ is transformed to a geometry with the barrier ending in the origin.

from rest for three values of $p$ are given in section IV. All three experiments concern the spin-up from 0 to $0.24 \mathrm{rad} / \mathrm{s}$ in a tank with radius $a=46.1 \mathrm{~cm}$, filled with water to a depth $H$ of $20 \mathrm{~cm}$. Thus, the theoretical ${ }^{1}$ spin-up time scale $H / \sqrt{\Omega \nu}$ is approximately $408 \mathrm{~s}$. The experiments show that immediately after the angular velocity is increased, a cyclonic vortex is formed at the end of the barrier. In section V, a simple analytical model for the trajectory and circulation of this cyclonic vortex is derived, and compared with measurements of the vortex trajectory from dye visualizations.

\section{EXPERIMENTAL SET-UP}

The spin-up experiments were performed by using a tank filled with tap water, placed on a rotating table. At $t=0$, the angular velocity of the table was suddenly increased from $\Omega-\Delta \Omega$ to $\Omega$, and was thereafter kept at this value during the experiment. It was verified that fluctuations in the final angular velocity are very small compared with the final angular velocity used in the experiments.

Quantitative results were obtained with small tracer particles floating at the surface of the fluid. Dye was added to the water in order to increase the contrast between the fluid and the particles. First, a video recording of the flow was made with a video camera corotating with the tank. Then, after the experiment, the recording was processed by a PC equipped with a frame grabber. For this purpose, an adapted version of the DigImage system developed by Dalziel ${ }^{12}$ was used. This is an image processing system that allows the tracking of particles based on a number of user-defined criteria such as brightness and size. Further processing of the particle trajectory data was enabled by an extension to the DigImage software developed by Van der Plas. ${ }^{13}$ This option provides the possibility to extract data files containing the particle velocities from the data file created by the particle tracking routine. The vorticity was obtained by matching the data with spline functions and manipulating the coefficients of this expansion. The stream function was calculated from the vorticity by using a Poisson solver. In this way the stream function of the solenoidal component of the velocity field is calculated, which is more elegant than applying integration techniques if the flow is not exactly divergence free.

In addition to the experiments with particles, experiments were performed with a small amount of dye added to the otherwise clear tap water. In this way, a qualitative im- pression of the flow field could be obtained. In every experiment described in this paper, the flow was seen to become turbulent shortly after the increase in angular velocity of the tank. However, the flow always laminarized early in the experiment. By adding dye at different stages, it was verified that the flow remained approximately two-dimensional after the flow had become laminar.

\section{STARTING FLOW}

The flow immediately after the impulsive increase in angular velocity is characterized by a uniform vorticity $-2 \Omega$ in a system corotating with the tank. Since the vorticity is related to the stream function by

$$
\omega=-\nabla^{2} \psi
$$

this leads to a Poisson equation for the stream function $\psi$ of the flow relative to the rotating tank:

$$
\nabla^{2} \psi=2 \Omega \text {. }
$$

Because of the zero normal flow condition, the sidewall boundary coincides with a streamline, the stream function of which is taken to be zero.

In this section we present analytical solutions of (2). For $p=-1$ and $p=0$, the solutions are simpler than for arbitrary values for $p$, and have been described before by Van Heijst, ${ }^{5}$ albeit in a slightly different way. In view of its further use in this paper, the solution for $p=0$ is derived here; the solution for $p=-1$ is presented without proof. For $p=1$ the barrier vanishes, yielding a purely circular geometry with a trivial solution for the stream function. The problem for arbitrary barrier length can also be treated analytically to a certain extent, but leads to a difficult integral expression for the expansion coefficients appearing in the series solution. Moreover, the solution is obtained in transformed coordinates with the barrier ending in the center of the tank. The integral expression and the transformation to the original geometry have been calculated numerically.

\section{A. $p=0$}

For $p=0$, the barrier reaches to the center of the tank. The problem for $\psi$ is stated in polar coordinates by

$$
\frac{\partial^{2} \psi}{\partial r^{2}}+\frac{1}{r} \frac{\partial \psi}{\partial r}+\frac{1}{r^{2}} \frac{\partial^{2} \psi}{\partial \theta^{2}}=2 \Delta \Omega,
$$

with $\psi=0$ at all parts of the boundary. The problem is solved by splitting the solution in a particular solution and a homogeneous solution:

$$
\psi(r, \theta)=\psi_{\text {part }}(r, \theta)+\psi_{\text {hom }}(r, \theta),
$$

with $\psi_{\text {part }}$ taking away the inhomogeneous right-hand side of (3), and $\psi_{\text {hom }}$ the solution of the resulting Laplace equation for $\psi-\psi_{\text {part }}$. Since $\psi_{\text {part }}$ is taken to be non-zero on a part of the boundary, the problem for $\psi_{\text {hom }}$ has Dirichlet boundary conditions. A convenient particular solution of (3) is

$$
\psi_{\text {part }}=\Delta \Omega r^{2} \sin ^{2} \theta .
$$

This solution vanishes at the barrier, and is equal to $\Delta \Omega a^{2} \sin ^{2} \theta$ at the circular part of the boundary. Thus, the problem for $\psi_{\text {hom }}$ becomes 


$$
\frac{\partial^{2} \psi_{\mathrm{hom}}}{\partial r^{2}}+\frac{1}{r} \frac{\partial \psi_{\mathrm{hom}}}{\partial r}+\frac{1}{r^{2}} \frac{\partial^{2} \psi_{\mathrm{hom}}}{\partial \theta^{2}}=0,
$$

with $\psi_{\text {hom }}(r, 0)=\psi_{\text {hom }}(r, 2 \pi)=0$, and $\psi_{\text {hom }}(a, \theta)=-\Delta \Omega a^{2}$ $\times \sin ^{2} \theta$. This equation is easily solved by separation of variables. Taking into account the boundary conditions for $\theta$ and the constraint that $\psi$ has to be zero at $r=0$, one finds that

$$
\psi_{\mathrm{hom}}=\Delta \Omega a^{2} \sum_{n=0}^{\infty} A_{n}\left(\frac{r}{a}\right)^{n+1 / 2} \sin \left(n+\frac{1}{2}\right) \theta,
$$

so that at $r=a$,

$$
-\sin ^{2} \theta=\sum_{n=0}^{\infty} A_{n} \sin \left(n+\frac{1}{2}\right) \theta \text {. }
$$

Application of the Fourier expansion of $\sin ^{2} \theta$ on the interval $[0,2 \pi]$ given by

$$
\sin ^{2} \theta=-\frac{4}{\pi} \sum_{n=0}^{\infty} \frac{\sin \left(n+\frac{1}{2}\right) \theta}{\left(n-\frac{3}{2}\right)\left(n+\frac{1}{2}\right)\left(n+\frac{3}{2}\right)},
$$

yields the coefficients $A_{n}$, so that

$$
\frac{\psi(r, \theta)}{\Delta \Omega a^{2}}=r^{\prime 2} \sin ^{2} \theta+\frac{4}{\pi} \sum_{n=0}^{\infty} \frac{r^{\prime n+1 / 2} \sin \left(n+\frac{1}{2}\right) \theta}{\left(n-\frac{3}{2}\right)\left(n+\frac{1}{2}\right)\left(n+\frac{3}{2}\right)},
$$

where $r^{\prime}=r / a$. The expression is an attractive representation of the solution, since the series converges rapidly, and can be easily evaluated on a computer; moreover, the explicit appearance of powers of $r^{\prime}$ may be used to obtain a lowest order solution in the vicinity of $r=0$. However, in this case the series can also be summed analytically. By introducing the complex variable $z^{\prime}=r^{\prime} \exp i \theta$, (10) can be written as

$$
\frac{\psi(r, \theta)}{\Delta \Omega a^{2}}=r^{\prime 2} \sin ^{2} \theta+\frac{4}{\pi} \operatorname{Im} \sum_{n=0}^{\infty} \frac{z^{\prime n+1 / 2}}{\left(n-\frac{3}{2}\right)\left(n+\frac{1}{2}\right)\left(n+\frac{3}{2}\right)} .
$$

The term of the series expression can be written as a sum of partial fractions, which can be summed by a straightforward procedure:

$$
\begin{aligned}
& \sum_{n=0}^{\infty} \frac{z^{\prime n+1 / 2}}{\left(n-\frac{3}{2}\right)\left(n+\frac{1}{2}\right)\left(n+\frac{3}{2}\right)} \\
& \quad=-\frac{3+z^{\prime}+z^{\prime 2}+3 z^{\prime 3}}{12 z^{\prime} \sqrt{z^{\prime}}}+\frac{\left(1-z^{\prime 2}\right)^{2}}{8 z^{\prime 2}} \ln \left(\frac{1+\sqrt{z^{\prime}}}{1-\sqrt{z^{\prime}}}\right) .
\end{aligned}
$$

Evaluation of the imaginary part of this expression in $r^{\prime}$ and $\theta$ leads to

$$
\begin{aligned}
\frac{\psi\left(r^{\prime}, \theta\right)}{\Delta \Omega a^{2}}= & r^{\prime 2} \sin ^{2} \theta+\frac{1}{\pi}\left[\frac{\sin \frac{3}{2} \theta+\frac{1}{3} r^{\prime} \sin \frac{1}{2} \theta-\frac{1}{3} r^{\prime 2} \sin \frac{1}{2} \theta-r^{\prime 3} \sin \frac{3}{2} \theta}{r^{\prime} \sqrt{r^{\prime}}}-\frac{1}{4} \sin 2 \theta\left(r^{\prime-2}-r^{\prime 2}\right)\right. \\
& \left.\times \ln \left(\frac{1+2 \sqrt{r^{\prime}} \cos \frac{1}{2} \theta+r^{\prime}}{1-2 \sqrt{r^{\prime}} \cos \frac{1}{2} \theta+r^{\prime}}\right)+\frac{1}{2}\left(r^{\prime-2} \cos 2 \theta-2+r^{\prime 2} \cos 2 \theta\right) \arctan \left(\frac{2 \sqrt{r^{\prime}} \sin \frac{1}{2} \theta}{1-r^{\prime}}\right)\right] .
\end{aligned}
$$

The stream function according to (10) or (13) is presented in Fig. 2d.

\section{B. $p=-1$}

In this case the barrier divides the flow into two equivalent parts. The solution in the upper half of the domain can be found with the same method used for $p=0$. The result is

$$
\frac{\psi(r, \theta)}{\Delta \Omega a^{2}}=r^{\prime 2} \sin ^{2} \theta+\frac{1}{\pi} \sum_{n=0}^{\infty} \frac{r^{\prime n+1 / 2} \sin (2 n+1) \theta}{\left(n-\frac{1}{2}\right)\left(n+\frac{1}{2}\right)\left(n+\frac{3}{2}\right)} .
$$

The flow in the lower half of the domain is found after a rotation over $\pi$ rad around $r=0$. The series can be summed using a similar procedure as for $p=0$, leading to

$$
\begin{aligned}
\frac{\psi\left(r^{\prime}, \theta\right)}{\Delta \Omega a^{2}}= & r^{\prime 2} \sin ^{2} \theta+\frac{1}{\pi}\left[\left(r^{\prime-1}-r^{\prime}\right) \sin \theta\right. \\
& -\frac{1}{4}\left(r^{\prime-2}-r^{\prime 2}\right) \sin 2 \theta \ln \left(\frac{1+2 r^{\prime} \cos \theta+r^{\prime 2}}{1-2 r^{\prime} \cos \theta+r^{\prime 2}}\right) \\
& +\frac{1}{2}\left(r^{\prime-2} \cos 2 \theta-2\right. \\
& \left.\left.+r^{\prime 2} \cos 2 \theta\right) \arctan \left(\frac{2 r^{\prime} \sin \theta}{1-r^{\prime 2}}\right)\right]
\end{aligned}
$$

The stream function according to this solution is presented graphically in Fig. 2a.

\section{C. $p=1$}

In this case the radial barrier is absent, and the solution consists simply of a solid-body rotation with angular velocity $-\Delta \Omega$, and stream function

$$
\psi=\frac{1}{2} \Delta \Omega\left(r^{2}-a^{2}\right)
$$



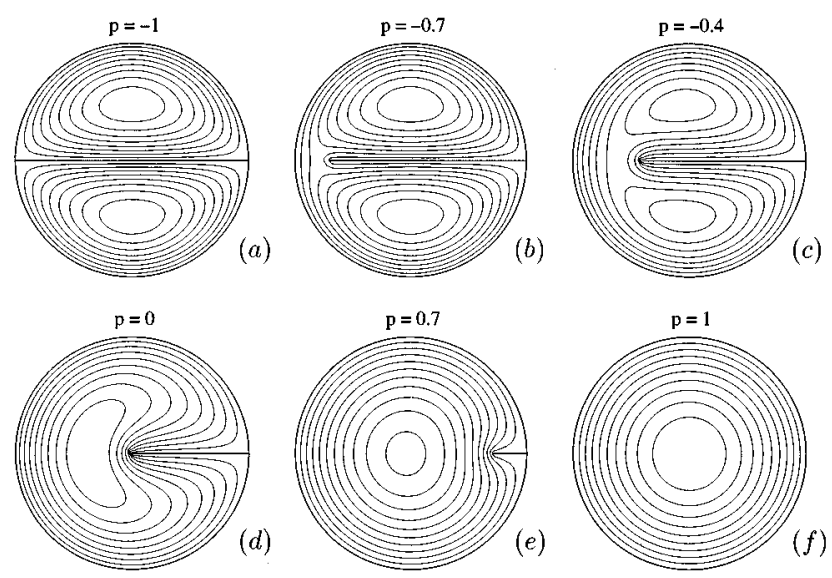

FIG. 2. Theoretical streamlines of the starting flow in a circular tank with a radial barrier. The parameter $p$ denotes the scaled $x$-coordinate of the end of the barrier.

\section{D. $-1<p<1$}

The problem in the geometry with the sharp edge at $(p a, 0)$ can be solved by using a conformal mapping to the geometry with the edge in the center. First, we take the particular solution,

$$
\psi_{\text {part }}=\Delta \Omega y^{2}=\Delta \Omega r^{2} \sin ^{2} \theta,
$$

so that for the homogeneous solution

$$
\nabla^{2} \psi_{\text {hom }}=0,
$$

with

$$
\psi_{\text {hom }}=0, \text { for } p<x / a<1 \wedge y=0,
$$

and

$$
\psi_{\text {hom }}=-\Delta \Omega a^{2} \sin ^{2} \theta, \text { for } r / a=1 \text {. }
$$

Since the homogeneous solution is a harmonic function, a conformal mapping may be used to transform the problem to the geometry with the barrier ending in the origin. The original geometry is described in the complex $z$-plane with $z=x+i y$, the transformed problem is described in the complex $w$-plane with $w=u+i v$, where $u, v$ and $w$ are dimensionless quantities; the radius of the circular part of the boundary in the $w$-plane is taken to be equal to one. This situation is illustrated in Fig. 1. The conformal mapping $w(z)$ is readily found as a Möbius transformation. By demanding that the vertices in the $w$-plane be mapped to the corresponding vertices in the $z$-plane, this mapping is fully determined, and is given by

$$
w=\frac{z / a-p}{1-p z / a},
$$

with inverse

$$
\frac{z}{a}=\frac{w+p}{1+p w} .
$$

The condition for a point $(u, v)$ at the boundary in the complex $w$-plane is found by the inverse transformation to the corresponding point in the $z$-plane, of which the value of the stream function is known to be $\Delta \Omega y^{2}$. Written in Cartesian coordinates it follows from (22) that

$$
\frac{x+i y}{a}=\frac{(u+p)(1+p u)+p v^{2}+i\left(1-p^{2}\right) v}{(1+p u)^{2}+p^{2} v^{2}},
$$

so that

$$
\frac{y}{a}=\frac{\left(1-p^{2}\right) v}{(1+p u)^{2}+p^{2} v^{2}} .
$$

For $u^{2}+v^{2}=1$ this becomes

$$
\frac{y}{a}=\frac{\left(1-p^{2}\right) \sin \phi}{p^{2}+2 p \cos \phi+1}
$$

where $\phi$ is the azimuthal angle in the $w$-plane; the radial position in the $w$-plane will be denoted by $\rho$. The boundary condition at $\rho=1$ is given by

$$
\left.\psi_{\mathrm{hom}}\right|_{\rho=1}=\Delta \Omega y^{2}(\rho, \phi)=\Delta \Omega a^{2} \frac{\left(1-p^{2}\right)^{2} \sin ^{2} \phi}{\left(p^{2}+2 p \cos \phi+1\right)^{2}} .
$$

As in the problem with $p=0$, the homogeneous solution in the $w$-plane can be written as a series in $\sin \left(n+\frac{1}{2}\right) \phi$ and powers of $\rho$ :

$$
\psi_{\mathrm{hom}}=\Delta \Omega a^{2} \sum_{n=0}^{\infty} A_{n} \rho^{n+1 / 2} \sin \left(n+\frac{1}{2}\right) \phi .
$$

In this equation, negative powers of $\rho$ have been omitted since they violate the condition of vanishing stream function at the edge. The coefficients $A_{n}$ are found by expanding the value of $\psi$ at $\rho=1$ to $\sin \left(n+\frac{1}{2}\right) \phi$. This leads to an integral expression for $A_{n}$, given by

$$
A_{n}=\frac{\left(1-p^{2}\right)^{2}}{\pi} \int_{0}^{2 \pi} \frac{\sin ^{2} \phi \sin \left(n+\frac{1}{2}\right) \phi}{\left(p^{2}+2 p \cos \phi+1\right)^{2}} d \phi .
$$

This integral has been evaluated numerically for a number of values for $p$. The results are presented together with the earlier results for $p=-1,0$ and 1 in Fig. 2. With this complement, Fig. 2 provides an overview of the full range of barrier lengths. If the barrier is absent $(p=1)$, the flow is circular (Fig. 2f). A small barrier (Fig. 2e) acts as a disturbance to this flow, affecting the flow in the vicinity of the barrier, but leaving the qualitative appearance of the flow far from the barrier unchanged. As the length of the barrier increases, the disturbance becomes noticeable in a larger part of the domain. For the negative values for $p$ presented in Figs. $2 b$ and c, the stream function has two extrema, implying the presence of a hyperbolic stagnation point at the negative $y$-axis. Further numerical calculations indicate that the splitting of the single cell into a flow with two stream function extrema occurs close to $p=-0.1$. 


\section{F. Kinetic energy of the starting flow}

The kinetic energy of the flow is defined as

$$
\begin{aligned}
T & =\frac{1}{2} \rho \int \mathbf{u} \cdot \mathbf{u} d A=\frac{1}{2} \rho \int\left[\left(\frac{\partial \psi}{\partial y}\right)^{2}+\left(\frac{\partial \psi}{\partial x}\right)^{2}\right] d A \\
& =\frac{1}{2} \rho \int \nabla \psi \cdot \nabla \psi d A,
\end{aligned}
$$

with $\rho$ the mass of the fluid per unit of area, and the integral taken over the entire fluid domain. Integration by parts of the third integral leads to

$$
T=\frac{1}{2} \rho \int \nabla(\psi \nabla \psi) d A-\frac{1}{2} \rho \int \psi \nabla^{2} \psi d A .
$$

With Gauss' theorem one can write the first term on the right-hand side as

$$
\frac{1}{2} \rho \int \nabla(\psi \nabla \psi) d A=\frac{1}{2} \rho \int(\psi \nabla \psi \cdot \mathbf{n}) d s,
$$

with $s$ the length of a line element of the boundary, and $\mathbf{n}$ the normal of the boundary curve. For flows with zero normal velocity at all parts of the boundary, this integral vanishes identically. In the second term on the right-hand side of (30), $\nabla^{2} \psi$ can be replaced by $-\omega$, so the expression for $T$ becomes

$$
T=\frac{1}{2} \rho \int \psi \omega d A .
$$

For the starting flow with uniform vorticity $-2 \Delta \Omega$ this becomes

$$
T=-\Delta \Omega \rho \int \psi d A .
$$

One can see from (33) that the energy of the starting flow on a bounded domain is always finite. Since the velocity at the end of a barrier is singular, this is not immediately clear from (29). However, the same conclusion would be drawn from an integration of $\mathbf{u} \cdot \mathbf{u}$ over a neighborhood of the end of the barrier. The integral expression given by (33) has been evaluated numerically for a number of values for $p$; the results are presented in Fig. 3. The data show that the kinetic energy decreases when the length of the barrier is increased. This leads to the conception that a division of the domain into smaller parts leads to lower relative velocities, and therefore to a lower energy.

\section{EXPERIMENTAL RESULTS}

\section{A. $p=0.78$}

The results of this experiment are presented in Fig. 4. The streamlines at $t=0$ show a close resemblance to the theoretical solution in Fig. 2e $(p=0.7)$; the flow is still approximately circular, with a small disturbance near the barrier. Shortly after the start of the experiment, a small cyclonic vortex is shed from the barrier; this is clearly visible in the results for $t=2 \mathrm{~s}$. However, this vortex is weak, and quickly disappears in the turbulent band of positive vorticity

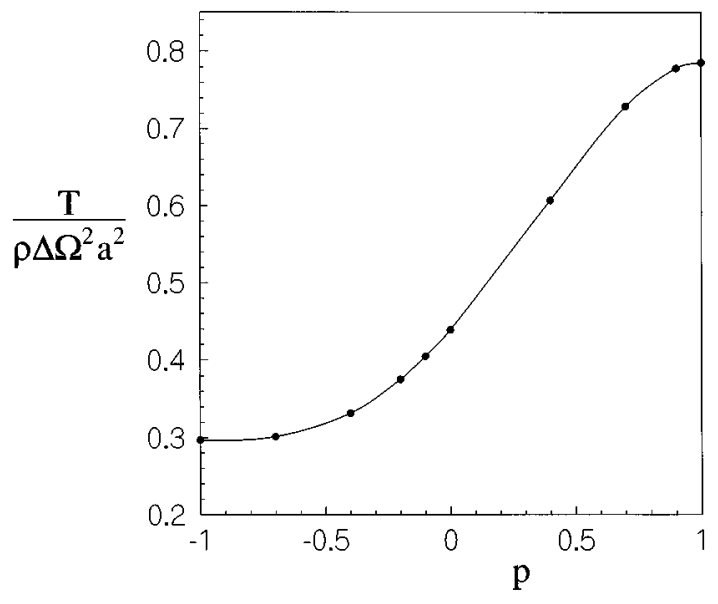

FIG. 3. Kinetic energy of the starting flow versus $p$.

at the sidewall of the tank. From $t=240 \mathrm{~s}$, the flow appears as a single cell, shifted slightly to the left to avoid the part of the tank that is 'shielded' by the barrier.

The graphs of $\omega$ versus $\psi$ provide information of the structure of the main anticyclonic vortex that is difficult to perceive from the stream function and vorticity contours only. First, the scatter in the $\omega(\psi)$-profiles is a measure of the instationarity of the flow. The observation that in this experiment, the data points in the $\omega, \psi$-plane are lying on an increasingly well-defined curve indicates that the flow gradually becomes steadier. Second, the shape of the $\omega(\psi)$-profiles indicates details of the spin-up mechanism. For instance, the shrinking of the part of the domain where the vorticity has kept its initial value $-2 \Omega$ and the transition of the upward curvature at $240 \mathrm{~s}$ to the downward curvature at $960 \mathrm{~s}$ are features that are connected with a weak radial flow driven by the Ekman layers. This secondary flow leads to the stretching and radial advection of vortex tubes, which generally is the main cause of the actual spin-up of the fluid. This issue is discussed further by Van de Konijnenberg and Van Heijst. ${ }^{5}$

The effect of the radial barrier with respect to the flow in a circular tank can be summarized as follows. First, the barrier appears to shield a part of the domain, causing a shift in the position of the anticyclonic cell. Second, the separation from the barrier leads to a stronger and more persistent turbulence than in comparable experiments in a circular tank without a barrier. ${ }^{5,10}$ Thus, the vorticity profile in the outer part of the tank is more strongly smoothed, and the spin-up occurs slightly faster than without the barrier.

\section{B. $p=0$}

The experimental results for $p=0$ are presented in Fig. 5. This experiment can be considered as the standard experiment with a radial barrier, since for $p=0$ the geometry is compatible with the use of cylindrical coordinates, and it shows the typical separation from the sharp edge, with the formation of a strong cyclonic vortex at the downstream end of the barrier. This separation occurs so quickly that it is difficult to obtain experimental data from the unseparated 

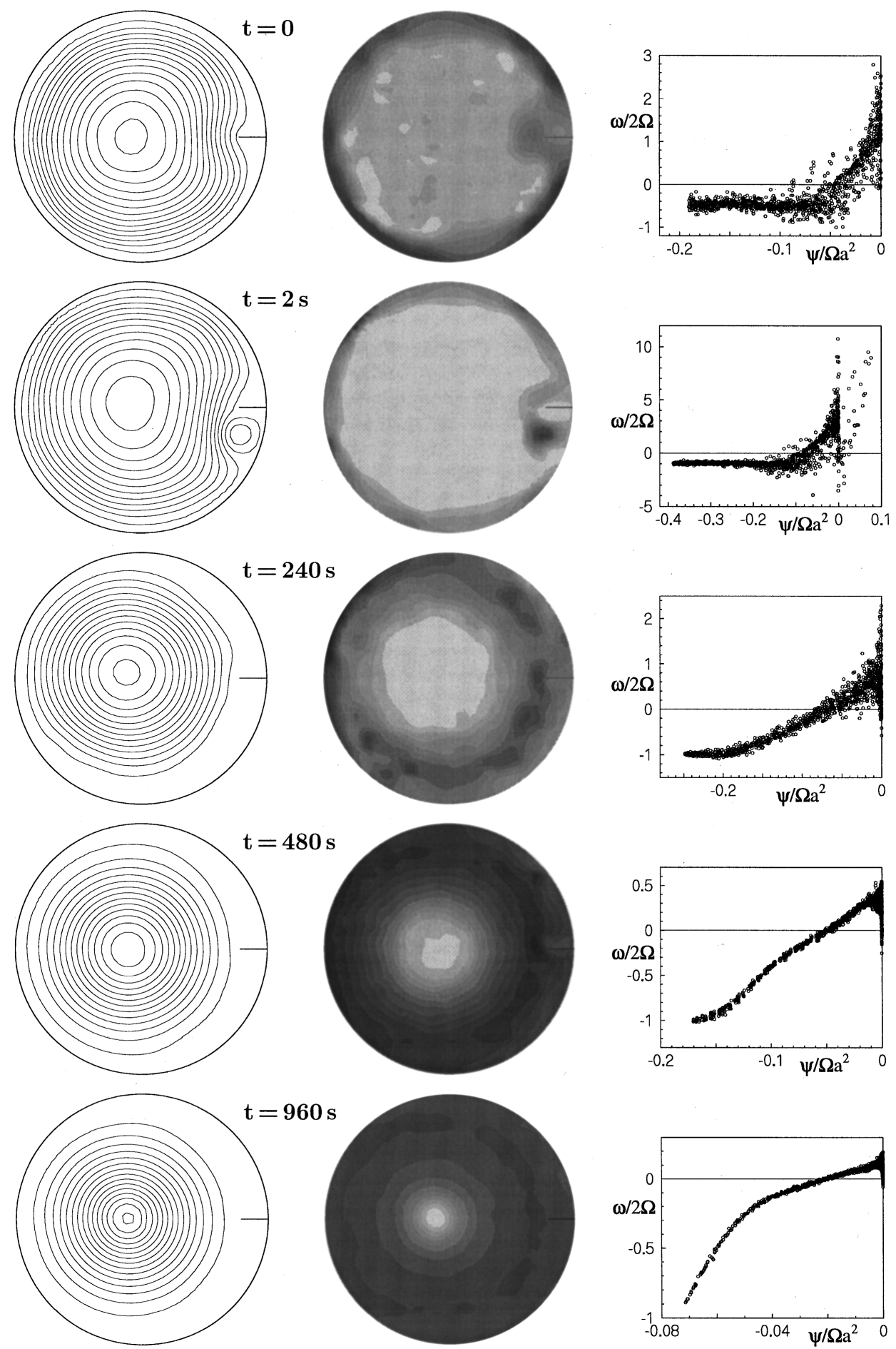

FIG. 4. Experimental results $[\psi, \omega$ and $\omega(\psi)]$ for spin-up from 0 to $0.24 \mathrm{rad} / \mathrm{s}$ in a circular tank with radius $46.1 \mathrm{~cm}$ and a radial barrier from the sidewall with length $10 \mathrm{~cm}(p=0.78)$. The tank has been filled to a depth of $20 \mathrm{~cm}$.

flow. Even in the results at " $t=0$ ", a small cyclonic vortexcan already be seen at the end of the barrier. These data were obtained within the time interval of about $1 \mathrm{~s}$ in which the rotating table accelerates from 0 to $0.24 \mathrm{rad} / \mathrm{s}$. One can see from the horizontal branch in the $\omega(\psi)$-graph that the relative vorticity has not yet reached its final initial value $-2 \Delta \Omega$.

Between $t=0$ and $t=30 \mathrm{~s}$, the cyclonic vortex grows in size and strength, and divides the initial anticyclonic region into two separate cells. The resulting pattern is then transformed into a four-cell structure (see Fig. 5 at $t=240 \mathrm{~s}$ ), which remains more of less stationary afterwards. The $\omega(\psi)$-graph at $t=30 \mathrm{~s}$ shows a strongly nonlinear branch corresponding to the cyclonic vortex that is shed from the radial barrier. This indicates that the cyclonic vorticity is concentrated in the center of the vortex, whereas the vorticity in the outer parts of this vortex is distributed more smoothly. This may be connected with the occurrence of turbulence in 

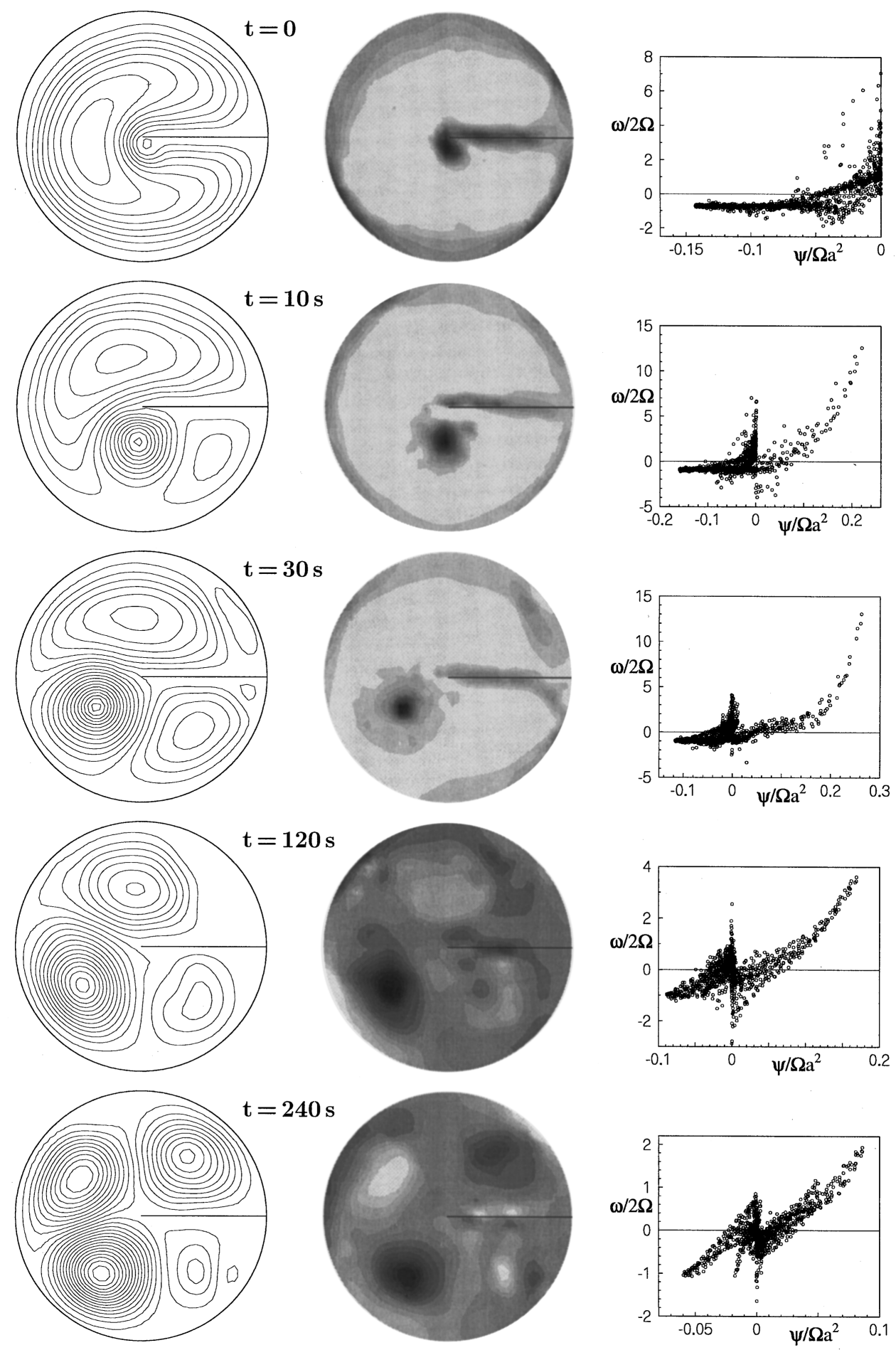

FIG. 5. Experimental results [ $\psi, \omega$ and $\omega(\psi)]$ for spin-up from 0 to $0.24 \mathrm{rad} / \mathrm{s}$ in a circular tank with radius $46.1 \mathrm{~cm}$ and a radial barrier from the sidewall to the center $(p=0)$. The tank has been filled to a depth of $20 \mathrm{~cm}$.

the outer parts of the vortex. Dye visualizations (see Fig. 9for a visualization in a comparable experiment) indicate that if the Reynolds number $L^{2} \Delta \Omega / \nu$ and Rossby number $\Delta \Omega / \Omega$ are sufficiently high, the shear layer that separates from the end of the barrier is unstable, and becomes turbulent. This turbulence will lead to a strong mixing, and therefore to a homogenization of the vorticity in the outer layer of the cyclonic vortex. It seems likely that this is the reason why the branch in the $\omega(\psi)$-graph at $t=30 \mathrm{~s}$ corresponding to the cyclonic vortex is almost horizontal in the interval $0.06<\psi / \Omega a^{2}<0.16$. In later stages this turbulence disappears, and the evolution of the vorticity profile is determined mainly by Ekman pumping. The nonlinearity of this mechanism will lead to a fast decay of strongly positive vorticity, so that the $\omega(\psi)$-profile gradually loses its strong curvature.

C. $p=-0.30$

This experiment, the results of which are presented in 

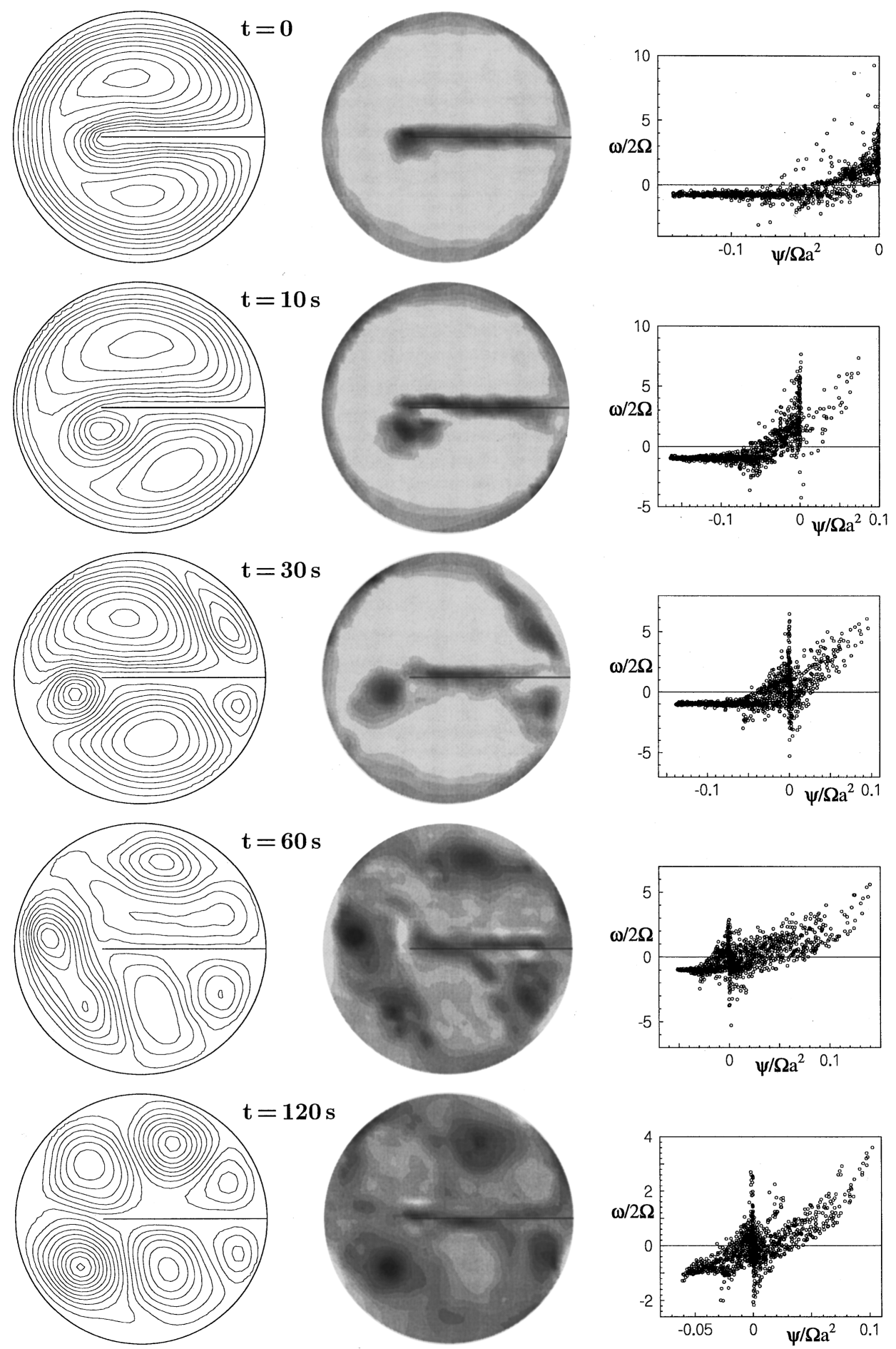

FIG. 6. Experimental results [ $\psi, \omega$ and $\omega(\psi)]$ for spin-up from 0 to $0.24 \mathrm{rad} / \mathrm{s}$ in a circular tank with radius $46.1 \mathrm{~cm}$ and a radial barrier from the sidewall with length $60 \mathrm{~cm}(p=-0.30)$. The tank has been filled to a depth of $20 \mathrm{~cm}$.

Fig. 6, exhibits some of the properties of the geometry withthe radial barrier extending to the other side of the tank $(p=-1)$. As predicted in section III, the stream function of the starting flow has two minima. Immediately after $t=0$, the flow separates from the end of the barrier, but the cyclonic cell does not become as strong as in the corresponding experiment with $p=0$. This may be caused by the flow around the barrier being weaker (see Fig. 8), and the cyclonic vortex becoming pinched between the end of the barrier and the circular part of the boundary. At the right side of the tank, the flow separates from the circular part of the boundary, leading to cyclonic vortices (see Fig. 6 at $t=30 \mathrm{~s}$ ). The flow then goes through a stage of complicated vortex interactions, ultimately leading to a pattern of six counterrotating vortices (see Fig. 6 at $t=120 \mathrm{~s}$ ). This pattern is not quite steady; small vortices are being formed continuously at the end of the barrier. These vortices interfere with the existing vortex pattern in the tank, and may disturb the streamline pattern. 
However, the qualitative appearance of the flow remains comparable with the flow at $120 \mathrm{~s}$ at all later times.

\section{VORTEX SHEDDING FROM THE BARRIER}

In section IV it was seen that the early phase of the experiments with a radial barrier is characterized by the shedding of a cyclonic vortex from the end of the barrier. In this section, an adapted version of a point-vortex model for vortex shedding by Brown and Michael ${ }^{14}$ and Rott ${ }^{15}$ is described and compared with the experimental results. Since the cyclonic vortex is very small in the beginning of the experiment, it is difficult to obtain accurate quantitative data by tracking particles. However, the position of the center of the vortex can be tracked easily with a dye visualization. Data obtained with this method have been used to validate the analytical model.

\section{A. Theoretical considerations}

The stream function of the starting flow is given by

$$
\begin{aligned}
\psi(r, \theta)= & \Delta \Omega r^{2} \sin ^{2} \theta \\
& +\Delta \Omega a^{2} \frac{4}{\pi} \sum_{n=0}^{\infty}\left(\frac{r}{a}\right)^{n+1 / 2} \frac{\sin \left(n+\frac{1}{2}\right) \theta}{\left(n-\frac{3}{2}\right)\left(n+\frac{1}{2}\right)\left(n+\frac{3}{2}\right)} .
\end{aligned}
$$

In the vicinity of the sharp edge, the leading term is

$$
\psi=-\frac{32 \Delta \Omega a^{2}}{15 \pi} \sqrt{\frac{r}{a}} \sin \frac{1}{2} \theta
$$

which corresponds to a potential flow around a semi-infinite plate, with velocity potential

$$
\phi=-\frac{32 \Delta \Omega a^{2}}{15 \pi} \sqrt{\frac{r}{a}} \cos \frac{1}{2} \theta
$$

In terms of the complex velocity potential

$$
\Phi=\phi+i \psi
$$

and complex position $z=x+i y$, this becomes

$$
\Phi=-\frac{32 \Delta \Omega a^{2}}{15 \pi} \sqrt{\frac{z}{a}} .
$$

The shed vortex is represented by a potential vortex at position $z_{0}$ with circulation $\Gamma$ and complex velocity potential

$$
\Phi=\frac{\Gamma}{2 \pi i} \ln \left(\frac{z}{a}-\frac{z_{0}}{a}\right) .
$$

The $z$-plane with a branch cut along the real positive axis can be transformed to the complex $\zeta$-plane with $\operatorname{Im} \zeta>0$ by the conformal mapping

$$
\zeta=\sqrt{\frac{z}{a}}
$$

Through this conformal mapping, the flow around the semiinfinite plate is transformed into a uniform flow in the $\zeta$-plane, and the potential vortex is transformed into a vortex with circulation $\Gamma$ in $\zeta_{0}=\sqrt{z_{0} / a}$. The complex velocity po- tential $\Phi_{\zeta}$ in the $\zeta$-plane consists of a uniform flow resulting from (38), plus the flow due to a potential vortex at $\zeta_{0}$ and its image vortex in $\bar{\zeta}_{0}$ :

$$
\Phi_{\zeta}=-\frac{32 \Delta \Omega a^{2}}{15 \pi} \zeta+\frac{\Gamma}{2 \pi i} \ln \left(\zeta-\zeta_{0}\right)-\frac{\Gamma}{2 \pi i} \ln \left(\zeta-\bar{\zeta}_{0}\right)
$$

In the $z$-plane this becomes

$$
\begin{aligned}
\Phi= & -\frac{32 \Delta \Omega a^{2}}{15 \pi} \sqrt{\frac{z}{a}}+\frac{\Gamma}{2 \pi i} \ln \left(\sqrt{\frac{z}{a}}-\sqrt{\frac{z_{0}}{a}}\right) \\
& -\frac{\Gamma}{2 \pi i} \ln \left(\sqrt{\frac{z}{a}}-\sqrt{\frac{\overline{z_{0}}}{a}}\right) .
\end{aligned}
$$

In this equation, $\sqrt{\overline{z_{0}} / a}$ should be interpreted as the scaled position of the transformed image in $\bar{\zeta}_{0}$. Since all regular points in the $z$-plane are transformed to the upper half plane of the $\zeta$-plane, this can only be achieved by a doubling of the $z$-plane to $0 \leqslant \theta \leqslant 4 \pi$; in this way every point in the $\zeta$-plane has its unique image in the $z$-plane. An expression for $\Gamma$ is found by imposing the Kutta condition, which implies that the velocity is zero in $\zeta=0$ or, consequently, that the velocity is finite in $z=0$. In the $z$-plane, the velocity field is given by

$$
\begin{aligned}
u-i v= & \frac{d \Phi}{d z}=-\frac{16 \Delta \Omega a}{15 \pi} \frac{1}{\sqrt{z^{\prime}}}+\frac{\Gamma}{4 \pi a i} \frac{1}{\sqrt{z^{\prime}}} \frac{1}{\sqrt{z^{\prime}}-\sqrt{z_{0}^{\prime}}} \\
& -\frac{\Gamma}{4 \pi a i} \frac{1}{\sqrt{z^{\prime}}} \frac{1}{\sqrt{z^{\prime}}-\sqrt{\overline{z_{0}}}}
\end{aligned}
$$

where the prime denotes a division by $a$. The singularity in $z=0$ is removed by taking

$$
\Gamma=\frac{64 \Delta \Omega a^{2} i}{15} \frac{\sqrt{z_{0}^{\prime}} \sqrt{\overline{z_{0}^{\prime}}}}{\sqrt{z_{0}^{\prime}}-\sqrt{\overline{z_{0}^{\prime}}}}=\frac{64 \Delta \Omega a^{2} i}{15} \frac{\zeta_{0} \bar{\zeta}_{0}}{\zeta_{0}-\bar{\zeta}_{0}}
$$

The velocity potential can be written as

$$
\begin{aligned}
\Phi= & -\frac{32 \Delta \Omega a^{2}}{15 \pi} \sqrt{z^{\prime}}+\frac{\Gamma}{2 \pi i} \ln \left(z^{\prime}-z_{0}^{\prime}\right) \\
& -\frac{\Gamma}{2 \pi i} \ln \left(\sqrt{z^{\prime}}+\sqrt{z_{0}^{\prime}}\right)-\frac{\Gamma}{2 \pi i} \ln \left(\sqrt{z^{\prime}}-\sqrt{\bar{z}_{0}^{\prime}}\right) .
\end{aligned}
$$

The term proportional to $\ln \left(z^{\prime}-z_{0}^{\prime}\right)$ corresponds to a potential vortex in $z_{0}$. Since a potential vortex does not induce any velocity in itself, this term can be omitted for determining the velocity in $z_{0}$. The velocity in $z_{0}$ therefore becomes 


\begin{tabular}{|c|c|c|c|}
\hline exp. & $\Omega-\Delta \Omega$ & $\Omega$ & $\Delta \Omega / \Omega$ \\
\hline- & $\mathrm{rad} / \mathrm{s}$ & $\mathrm{rad} / \mathrm{s}$ & - \\
\hline \hline 1 & 0 & 0.13 & 1 \\
2 & 0.13 & 0.25 & 0.52 \\
3 & 0.25 & 0.37 & 0.32 \\
4 & 0.37 & 0.47 & 0.21 \\
\hline
\end{tabular}

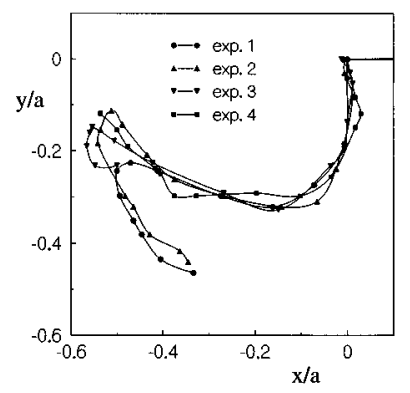

FIG. 7. Experimental results for the trajectory of the center of the shed vortex for $p=0$.

$$
\begin{aligned}
\frac{d \overline{z_{0}}}{d t}=\left.\frac{d \Phi}{d z}\right|_{z_{0}}= & -\frac{16 \Delta \Omega a^{2}}{15 \pi} \frac{1}{\sqrt{z_{0}^{\prime}}} \\
& -\frac{\Gamma}{4 \pi i} \frac{1}{\sqrt{z_{0}^{\prime}}}\left(\frac{1}{2 \sqrt{z_{0}^{\prime}}}+\frac{1}{\sqrt{z_{0}^{\prime}}-\sqrt{\overline{z_{0}^{\prime}}}}\right), \\
= & -\frac{16 \Delta \Omega a^{2}}{15 \pi} \frac{1}{\sqrt{z_{0}^{\prime}}}\left(1+\frac{\frac{1}{2} \sqrt{\overline{z_{0}^{\prime}}}}{\sqrt{z_{0}^{\prime}}-\sqrt{\overline{z_{0}^{\prime}}}}\right. \\
& \left.+\frac{\sqrt{z_{0}^{\prime}} \sqrt{\overline{z_{0}^{\prime}}}}{\left(\sqrt{z_{0}^{\prime}}-\sqrt{\overline{z_{0}^{\prime}}}\right)^{2}}\right) .
\end{aligned}
$$

Since there is no physical length scale in the flow around a semi-infinite plate, there is no curvature in the trajectory of the vortex, so $\theta$ must be constant. Thus, (47) is essentially an equation for $d r_{0} / d t$. Applying the condition that $d r_{0} / d t$ must be a real quantity leads to

$$
\begin{aligned}
& \theta=\frac{3}{2} \pi, \\
& \frac{r}{a}=\left(\frac{2 \sqrt{2} \Delta \Omega t}{5 \pi}\right)^{2 / 3},
\end{aligned}
$$

and

$$
\frac{\Gamma}{\Delta \Omega a^{2}}=\frac{32}{15}\left(\frac{8 \Delta \Omega t}{5 \pi}\right)^{1 / 3}
$$

\section{B. Discussion}

The point-vortex model is valid only for $r / a \ll 1$, that is, if the vortex in the experiment is still very small. Since quantitative data with floating particles are hard to obtain in this regime, four dye experiments have been performed with $p=0$, and different values for the initial angular velocity $\Omega-\Delta \Omega$. Before imposing the increase in angular velocity, dye was added at the upstream end of the barrier. After the start of the experiment, the dye is advected rapidly along the barrier, and detaches from the sidewall. Since some of the dye sticks to the barrier, the initial blob of dye is stretched to a thin filament (results of a similar visualization are presented in Fig. 9). This dye filament clearly shows the position of the center of the shed vortex. The trajectories of this center, as measured from the video screen, are presented in

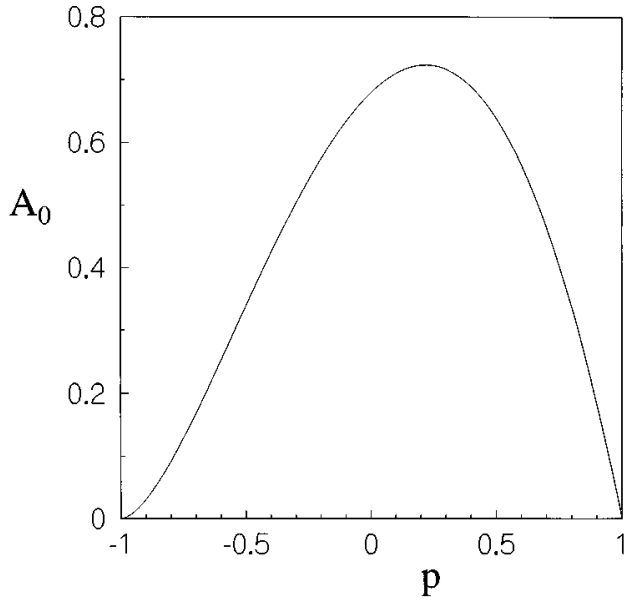

FIG. 8. The coefficient $A_{0}$ of the lowest-order term in the solution for the starting flow as a function of $p$, as calculated from (28).

Fig. 7. As according to the two-dimensional point-vortex model, the trajectories are similar for all four experiments. From the data points close to the end of the barrier, one can recognize the trajectory angle of $\frac{3}{2} \pi$ rad predicted by the point-vortex model. However, at $y / a=-0.2$ the vortex is deflected towards the negative $x$-direction. One can understand qualitatively why this deviation from the point-vortex model occurs. In the point-vortex model a potential flow around a semi-infinite plate is used. This implies that the velocity component in the $x$-direction decreases from the center along the negative $y$-axis, but always remains positive. However, according to the theoretical starting flow (see Fig. 2d) the velocity close to the intersection of the negative $y$-axis with the circular boundary is pointed to the left. In this respect, the lowest-order approximation for the velocity field is satisfactory for small $r / a$ only. Beyond this range, higherorder terms can no longer be ignored, and will cause a deflection of the vortex in the negative $x$-direction.

According to the point-vortex model, the crucial factor determining the growth rate of the shed vortex is the proportionality constant $A_{0}$ in the series expansion (10) for the stream function. One can also evaluate the corresponding constant $A_{0}$ for other values of $p$ by taking $n=0$ in (28). In Fig. 8, this quantity is presented as a function of $p$. According to this figure, $A_{0}$ is zero for $p=-1$ and $p=1$, which agrees with the absence of flow around a sharp edge in these cases. These data agree roughly with the relative strengths of the cyclonic vortices in the experiments. It is clear that eventually, the geometric constraints will affect the vortex as well, but in the beginning of the experiment, the flow close to the end of the barrier is determined by $A_{0}$ only.

\section{Instability of the shed vortex}

The point-vortex model gives a very schematic depiction of the actual flow field. In particular, the representation of the positive vorticity by a singularity in a single point is unrealistic. Moreover, the model does not describe the transport of positive vorticity from the end of the barrier to the center of the vortex. It is more realistic to picture the cy- 

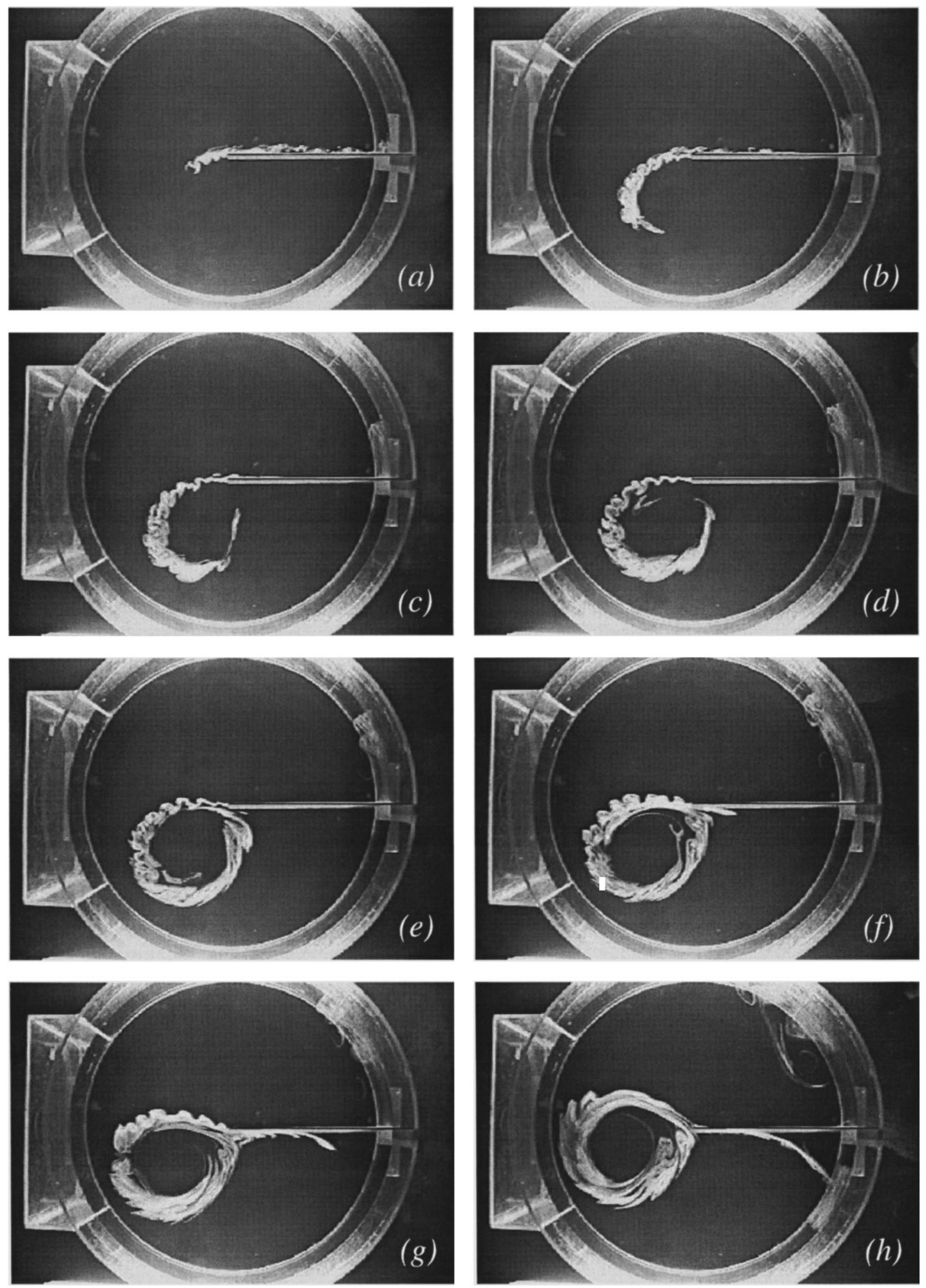

FIG. 9. Dye visualization of the flow instability in the outer region of the cyclonic vortex that is shed from the radial barrier. Experimental parameters: $\Omega=0.47 \mathrm{rad} / \mathrm{s} ; \Delta \Omega=0.10 \mathrm{rad} / \mathrm{s}$; tank radius $a=46.1 \mathrm{~cm}$, barrier length $46.1 \mathrm{~cm}(p=0)$. The pictures were taken at $25,30,35,40,45,50,60$ and $80 \mathrm{~s}$, respectively. The dye was added after $15 \mathrm{~s}$ at the upper right side of the barrier.

clonic vortex as a free shear layer, which is shed from the end of the barrier and curls up to a spiral. In this way one understands better why the vorticity distribution of the experimental vortex is diffuse, rather than concentrated in a single point. This shear layer can be observed experimentally by adding dye to the fluid. In all experiments described in this section, the shear layer shows a Kelvin-Helmholtz instability; in most experiments, this instability leads to threedimensional turbulence. A dye visualization of the KelvinHelmholtz instability in the shear layer is presented in Fig. 9. 
This experiment concerns the spin-up from 0.37 to 0.47 $\mathrm{rad} / \mathrm{s}$; due to the non-zero value of the initial angular velocity, the flow becomes stabilized with respect to vertical excursions, so that three-dimensional turbulence is suppressed for the most part. The dye was released at $15 \mathrm{~s}$ after the start of the experiment. At this time, the cyclonic vortex has reached a fair size, so that its center remains undyed. The Kelvin-Helmholtz instability shows up as the wavy deformation of the separated dye line; this is particularly clear in Figs. 9d and e. Note that Fig. 9 also gives a rough impression of the trajectory of the shed vortex.

\section{CONCLUSION}

In this paper, the spin-up in a circular tank with a radial inward barrier is investigated experimentally for three barrier lengths; moreover, analytical expressions for the flow immediately after the increase in angular velocity are presented. For small barrier length, the flow remains approximately azimuthal, and is similar to the flow in a circular tank without a barrier ${ }^{2-5}$ For longer barriers, the flow breaks up in a pattern of counterrotating vortices, confirming the general picture of spin-up in a tank with a flat bottom described by Van Heijst ${ }^{6}$ and Van Heijst, Davies and Davis. ${ }^{7}$ Immediately after the start of the experiment, a cyclonic vortex is shed from the end of the barrier. The trajectory of this vortex has been measured with a dye visialization. These experimental data have been compared with analytical results from a pointvortex model. In the vicinity of the end of the barrier, the flow field is dominated by one of its irrotational components, so that potential theory can be used to calculate the motion of the shed vortex. At a greater distance from the barrier end, the flow can no longer be considered as irrotational, and a more sophisticated model has to be used.

\section{ACKNOWLEDGMENT}

One of us (JvdK) gratefully acknowledges financial support from the Dutch Foundation of Fundamental Research (FOM).

${ }^{1}$ H.P. Greenspan and L.N. Howard, "On a time-dependent motion in a rotating fluid,' J. Fluid Mech. 17, 385 (1963).

${ }^{2}$ E.H. Wedemeyer, "The unsteady flow within a spinning cylinder," J. Fluid Mech. 20, 383 (1964).

${ }^{3}$ P.D. Weidman, "On the spin-up and spin-down of a rotating fluid,' J. Fluid Mech. 77, 685 (1976).

${ }^{4}$ J.M. Hyun, F. Leslie, W.W. Fowler, and A. Warn-Varnas, "Numerical solutions for spin-up from rest in a cylinder,' J. Fluid Mech. 127, 263 (1983).

${ }^{5}$ J.A. van de Konijnenberg and G.J.F. van Heijst, "Nonlinear spin-up in a circular cylinder,' Phys. Fluids 7, 2989 (1995).

${ }^{6}$ G.J.F. van Heijst, "Spin-up phenomena in non-axisymmetric containers," J. Fluid Mech. 206, 171 (1989).

${ }^{7}$ G.J.F. van Heijst, P.A. Davies, and R.G. Davis, "'Spin-up in a rectangular container,', Phys. Fluids A 2, 150 (1990).

${ }^{8}$ G.J.F. van Heijst, L.R.M. Maas, and C.W.M. Williams, "The spin-up of fluid in a rectangular container with a sloping bottom,'” J. Fluid Mech. 265, 125 (1994).

${ }^{9}$ Y.K. Suh, "Numerical study on two-dimensional spin-up in a rectangle," Phys. Fluids 6, 2333 (1994)

${ }^{10}$ J.A. van de Konijnenberg, "Spin-up in non-axisymmetric containers,", Ph.D. thesis, Eindhoven University of Technology, the Netherlands, 1995.

${ }^{11}$ H.I. Andersson, J.T. Billdal, and G.J.F. van Heijst, "Spin-up in a semicircular container,', Int. J. Num. Meth. in Fluids. 15, 503 (1992).

${ }^{12}$ S. Dalziel, 'DigImage. Image processing for fluid dynamics,' Cambridge Environmental Research Consultants, Ltd., 1992.

${ }^{13}$ G.A.J. van der Plas, "Introduction manual for particle tracking with DigImage,' Internal report No. R-1323-D, Faculty of Technical Physics, Fluid Dynamics Laboratory, Eindhoven University of Technology, the Netherlands, 1994.

${ }^{14}$ C.E. Brown and W.H. Michael, "Effect of leading edge separation on the lift of a delta wing,', J. Aeronaut. Sci. 21, 690 (1954).

${ }^{15}$ N. Rott, "Diffraction of a weak shock with vortex generation,' J. Fluid Mech. 1, 111 (1956). 